\title{
Malignant myoepithelioma of the breast: A case report and review of the literature
}

\author{
MARIA PAPAZIAN $^{1 *}$, IOANNIS KALANTZIS ${ }^{2 *}$, GEORGIOS GALANOPOULOS $^{1}$, ILIANA MANI ${ }^{2}$, \\ OLYMPIA TZAIDA $^{1}$, IOANNA IACOVIDOU ${ }^{1}$ and NIKOLAOS ZIRAS ${ }^{2}$
}

Departments of ${ }^{1}$ Pathology and ${ }^{2}$ Oncology, 'METAXA' Anticancer Hospital, 18537 Piraeus, Greece

Received July 29, 2015; Accepted October 27, 2015

DOI: $10.3892 / \mathrm{mco} .2016 .808$

\begin{abstract}
Malignant myoepithelioma of the breast is an extremely rare tumor composed entirely or almost entirely of malignant spindle cells with myoepithelial differentiation. Only a limited number of case reports have been descibed to date; therefore the biological behavior and treatment outcomes of this rare tumor have not been clearly determined. Herein, we present a case of a 74-year-old woman who was admitted with inflammatory-like cancer of the breast, presenting with invasion of the chest wall and axillary lymph node metastasis at the time of diagnosis. The histological examination revealed a tumor composed of epithelioid and spindle cells with moderate to marked nuclear atypia, with foci of hemorrhage and necrosis. The tumor cells were immunoreactive for vimentin, p63, p53, CD10, cytokeratin (CK)8/18, CKAE1-3 and S-100. Finally, a diagnosis of myoepithelial carcinoma of the breast was established. Neoadjuvant chemotherapy was first administered and proved to be ineffective. Due to locoregional progression that was associated with the development of an abscess and subsequent excessive bleeding, a palliative mastectomy was performed. Postoperatively, one more cycle of systemic chemotherapy was administered. However, the patient experienced an early relapse to the chest wall and succumbed to septic shock due to persistent local infection. The aggressiveness and chemoresistance of the tumor in this case was consistent with the existing bibliography.
\end{abstract}

Correspondence to: Dr Maria Papazian, Department of Pathology, 'METAXA' Anticancer Hospital, 51 Mpotasi Street, 18537 Piraeus, Greece

E-mail: papazianmaria8@gmail.com

Dr Ioannis Kalantzis, Department of Oncology, 'METAXA' Anticancer Hospital, 51 Mpotasi Street, 18537 Piraeus, Greece

E-mail: johnkalantzis@hotmail.com

*Contributed equally

Key words: malignant myoepithelioma, myoepithelial carcinoma, breast carcinoma, chemotherapy, local recurrence, immunohistochemistry

\section{Introduction}

Myoepithelial cells display characteristics of epithelial as well as smooth muscle cells, and are usually located in the breast, throughout the mammary duct system, as an interrupted layer of stellate cells between the continuous luminal epithelial cell layer and the basement membrane $(1,2)$. Therefore, neoplasms that arise from myoepithelial cells exhibit both epithelial and smooth muscle cell characteristics, but lack ductal differentiation (3).

Despite the fact that myoepithelial cells are part of the structure of the human breast, pure myoepithelial neoplasms are extremely uncommon and the number of such reported case studies is limited (3-5).

Tavassoli (6) proposed that five types of lesions are derived from or are composed of a dominant to pure population of myoepithelial cells: Adenoid cystic carcinoma, a rare mixed tumor referred to as pleomorphic carcinoma, multifocal myoepitheliosis, adenomyoepithelioma and malignant myoepithelioma (myoepithelial carcinoma). The latter has a phenotype similar with that of metaplastic carcinoma (6-8).

Myoepithelial carcinoma is generally a solitary lesion, characterized by an infiltrating proliferation of plump atypical spindle cells with easily identifiable mitotic figures and without an epithelial component, dispersed in a collagenous stroma (1,8-10). Myoepithelial cells are usually prominent in ducts and ductules in the periphery of the lesion.

The diagnosis of malignancy is based on the presence of marked cellularity, high mitotic rate, ill-defined margins, infiltrative growth pattern, necrosis and local recurrence of the tumor $(4,9,11)$.

Isolated cases of myoepithelial carcinoma have been described arising from a preexisting adenomyoepithelioma (7).

\section{Case report}

A 74-year-old woman was admitted to our medical center with a firm mass on her left breast, sized $\sim 10 x 7 \mathrm{~cm}$, which was identified on routine physical examination. The entire breast was erythematous and warm, with a widespread edema of the skin and a widely ulcerated nipple, mimicking advanced inflammatory cancer.

The patient had a medical history of endometrial adenocarcinoma, endometrioid type (International Federation 
of Gynecology and Obstetrics stage IA, World Health Organization stage T1N0M0) treated with radical hysterectomy and pelvic radiotherapy (25 sessions of radiotherapy) 4 years prior (08/2009). Menarche occurred at the age of 13 years and menopause at 50 years. The patient reported four pregnancies (two live births and two abortions); she had never smoked or consumed alcohol on a regular basis and had never received exogenous hormones, contraceptives or any chronic drug treatment. There was no family history of breast cancer.

The laboratory tests and serum tumor marker levels were within the normal range. A bone scan revealed no bone metastasis. A computed tomography (CT) scan revealed multiple diffuse lesions in the left breast (Fig. 1A and B), as well as abnormally enlarged unilateral axillary lymph nodes, indicative of metastasis. No distant metastases were detected. Aspiration biopsy cytology was performed, confirming the diagnosis of breast cancer.

Due to the nature of the tumor (large size with invasion of the skin and the chest wall, with additional involvement of the axillary lymph nodes), the patient was staged as IIIb and considered inoperable. Thus, neoadjuvant chemotherapy was initiated, consisting of three cycles of docetaxel $\left(75 \mathrm{mg} / \mathrm{m}^{2}\right)$, doxorubicin $\left(50 \mathrm{mg} / \mathrm{m}^{2}\right)$ and cyclophosphamide $\left(500 \mathrm{mg} / \mathrm{m}^{2}\right)$, repeated every 3 weeks.

Follow-up CT scans of the patient after completion of therapy revealed disease progression, clearly indicating the aggressiveness of the tumor and unresponsiveness to treatment (Fig. 1C and D).

The surgical team performed drainage of an abscess that had developed in the tumor area; however, due to excessive bleeding, a palliative simple mastectomy was finally performed (Fig. 2).

Postoperatively and prior to the administration of further chemotherapy, the patient experienced early locoregional recurrence (Fig. 2), also indicative of the resistance of the tumor to treatment. First-line chemotherapy with weekly doses of paclitaxel $\left(70 \mathrm{mg} / \mathrm{m}^{2}\right)$, carboplatin (area under the curve 2) and bevacizumab $(10 \mathrm{mg} / \mathrm{kg})$ every 3 weeks was scheduled.

Unfortunately, only two weekly doses of chemotherapy were administered, as the patient developed a severe disease-related infection, leading to fatal septic shock. Consequently, the evaluation of the effectiveness of the administered regimens could not be completed.

Gross findings. The dimensions of the surgical specimen were $23 \times 15.5 \times 8 \mathrm{~cm}$, with skin attached to the exterior surface. On dissection, almost the entire breast was occupied by firm, multilobulated grayish-white areas measuring $16 \mathrm{~cm}$ in greater diameter. The cut surface was partially solid, with areas of cystic degeneration, hemorrhage and necrosis. The tumor infiltrated all the surgical margins, as well as the nipple (Fig. 3a).

Histopathology. The microscopic examination revealed an invasive proliferation (Fig. 3b) of spindle-shaped/sarcomatoid cells (Fig. 3c), as well as epithelioid cells. The latter type exhibited vesicular nuclei, prominent nucleoli, variable nuclear atypia and eosinophilic cytoplasm (Fig. 3d). The neoplastic cells were diffusely arranged in a reticular or storiform pattern, with several cystically dilated/pseudoangiomatous/alveolar spaces (Fig. 3e). The mitotic rate was up
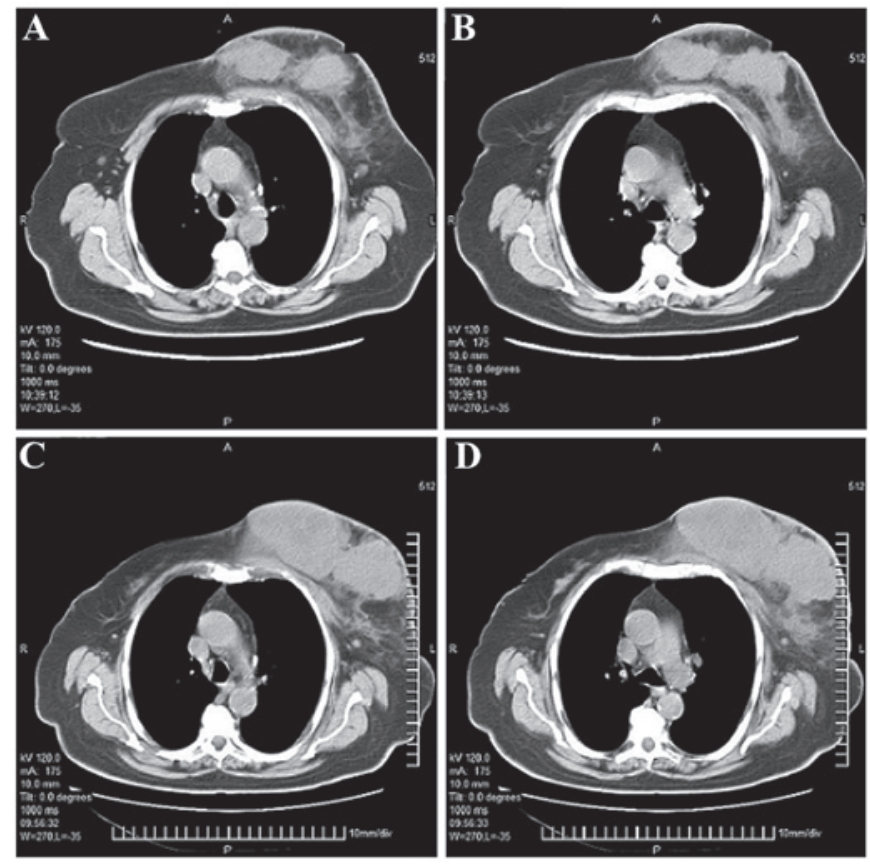

Figure 1. Computed tomography (CT) scans of the chest prior to and following neoadjuvant chemotherapy. (A and B) CT scans prior to the administration of therapy. (C and D) CT scans after 3 cycles of neoadjuvant chemotherapy with docetaxel, doxorubicin and cyclophosfamide reveal disease progression.
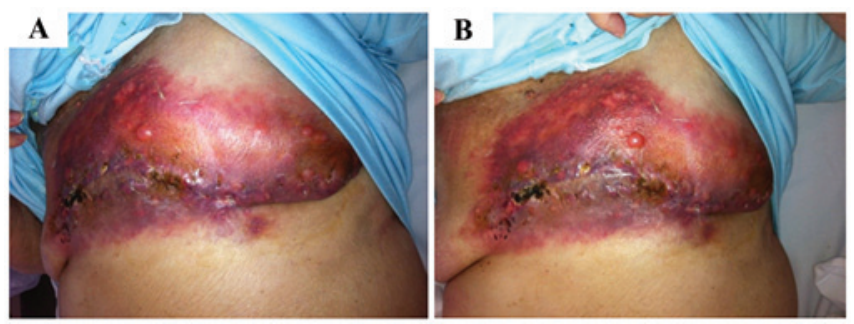

Figure 2. (A and B) Images of the patient after performing a palliative mastectomy and prior to the administration of first-line chemotherapy reveal locoregional recurrence of the tumor.

to 6 mitoses/10 high-power fields and the Ki- 67 proliferation index was $\sim 40 \%$.

Extensive areas of necrosis and hemorrhage, neoplastic infiltration of vessel walls, mild mixed inflammatory infiltrate, as well as entrapment of normal breast tissue (ducts and lobules) were detected within the tumor (Fig. 3f).

Immunohistochemistry (IHC). IHC analysis for cytokeratin (CK)AE1-3, CK5/6, CK7, CK14, CK8/18, CK20, epithelial membrane antigen, $\mathrm{S}-100$, smooth muscle actin (SMA), desmin, caldesmon, calponin, vimentin, mammoglobulin, human gross cystic disease fluid protein-15, estrogen receptor (ER), progesterone receptor (PR), MIB-1, p53, p63, topoisomerase IIa, human epidermal growth factor receptor 2 (HER2), CD10, CD31, CD34 and CD99 was performed.

The tumor cells were immunoreactive for vimentin, p63, p53, CD10, S-100, CK8/18 and CKAE1-3. Focal positivity for caldesmon and desmin (focal myoid transformation) was also observed. On the basis of the immunophenotype, the lesion was finally diagnosed as malignant myoepithelioma (Fig. 4). 

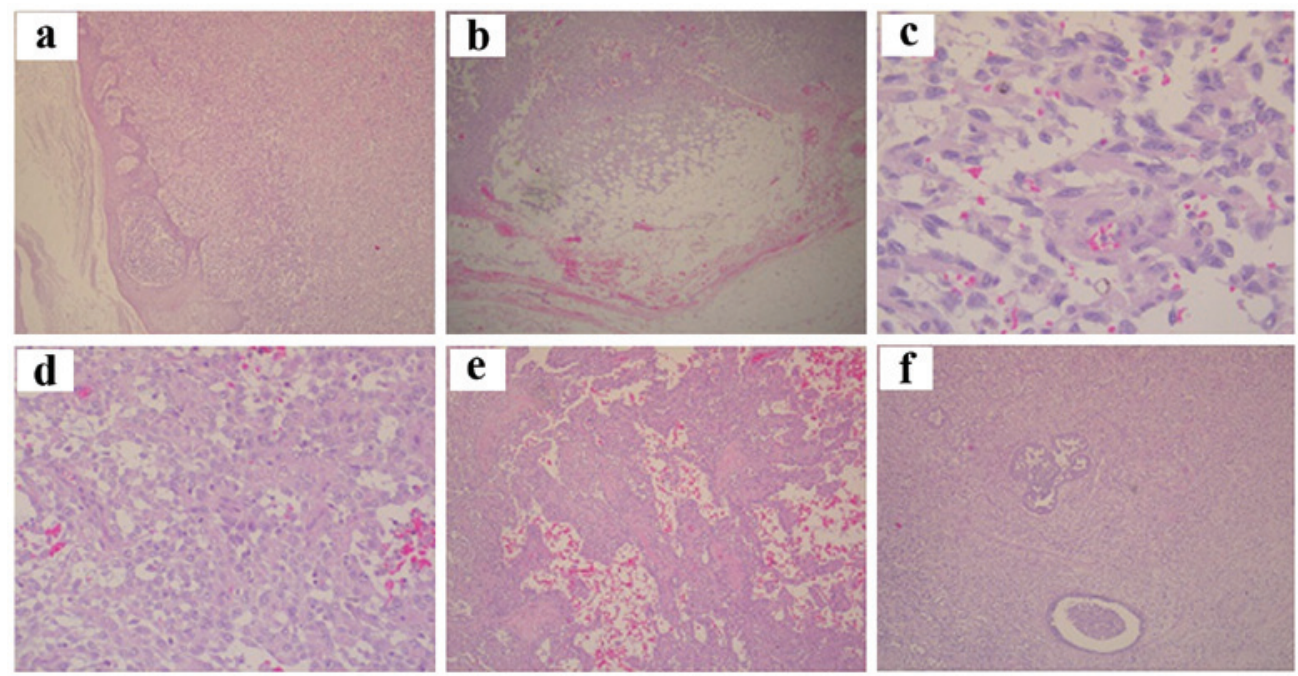

Figure 3. Hematoxylin and eosin staining. (a) Diffuse infiltration/ulceration of the nipple and skin (magnification, x50); (b) invasive proliferation, diffuse arrangement (magnification, x25); (c) spindle-shaped/sarcomatoid cells (magnification, x400); (d) epithelioid cells with vesicular nuclei and eosinophilic cytoplasm (magnification, x200); (e) reticular/storiform pattern with cystically dilated/pseudoangiomatous spaces (magnification, x100); and (f) solid growth pattern, with entrapment of normal breast tissue (magnification, $\mathrm{x} 100$ ).
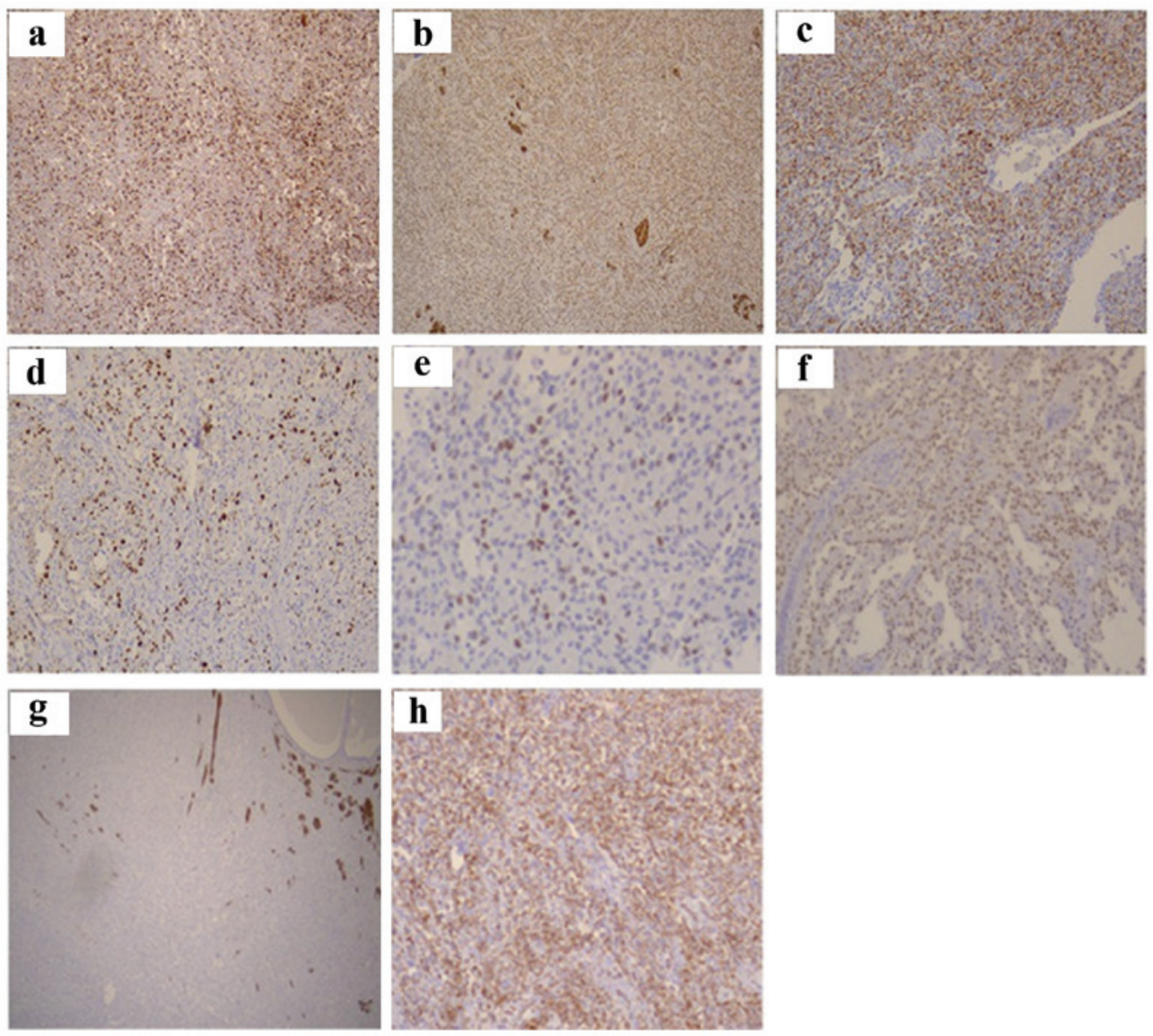

Figure 4. Immunohistochemistry for (a) S-100 (magnification, x100); (b) calponin (magnification, x100); (c) CK8/18 (magnification, x200); (d) Ki-67 (magnification, x100); (e) p53 (magnification, x400); (f) p63 (magnification, x400); (g) desmin (focal myoid differentiation; magnification, x50); and (h) vimentin (magnification, $\mathrm{x} 200)$. CK, cytokeratin.

\section{Discussion}

Myoepithelial carcinomas of the breast are extremely rare and represent a challenge in terms of diagnosis and treatment $(1,9,12)$. This type of neoplasm occurs in women aged 25-81 years (mean, 54 years) $(9,11)$. The tumor size ranges between 1.4 and $17 \mathrm{~cm}$ (mean, $3.5 \mathrm{~cm}$ ). The lesions are typically multinodular with central necrosis. Cytologically, 
the tumors are composed of epithelioid, plasmatocytoid and spindle cells (11-13).

In our case, the clinical manifestations (age 74 years and tumor size $16 \mathrm{~cm}$ ), the macroscopic findings (myltinodularity of the tumor with areas of central necrosis and hemorrhage) and the microscopic examination (biphasic pattern of growth with epithelioid and spindle cells entrapping normal breast tissue) contributed to the diagnosis of malignant myoepithelioma.

The contribution of the IHC results was key to confirming the diagnosis. The neoplastic cells exhibited immunoreactivity for markers indicative of myoepithelial differentiation (p63, p53 and S-100) and focal positivity for specific CKs (CK8/18, and CKAE1/AE3). The tumor cells also exhibited focal myoid differentiation, particularly around terminal ducts, which was highlighted by immunoreactivity for caldesmon and desmin. The tumor was also triple-negative (negative staining for ER, PR and HER2), with a Ki-67 proliferation index of $\sim 40 \%$.

Antibodies useful for detecting myoepithelial cells are directed against $\mathrm{CKs}$ and myofilaments. Antibodies against high-molecular weight CKs (CK5, CK5/6, CK14 and CK17) react in most lesions (3). Strong nuclear p63 and p53 immunostaining is observed, while S-100 is typically positive $(90 \%)$. There may be a positive reaction for muscle markers, such as calponin (86\%), muscle-specific actin, desmin (14\%) and SMA (36\%), as well as for basement membrane proteins, type IV collagen and laminin. Occasional cells exhibit immunostaining with glial fibrillary acidic protein (GFAP) $(5,7,9,11,14)$. All the tumors typically lack ER and PR receptors and are mitotically active $(14,15)$, purely composed of myoepithelial cells. Myoid transformation is most frequently encountered around terminal ducts and lobules, in the absence of epithelial proliferation $(7,8)$.

The differential diagnosis may be challenging, particularly when it comes to spindle cell/metaplastic carcinomas or the rare primary spindle cell sarcomas of the breast. Occasionally, these tumors are almost impossible to differentiate without IHC evaluation (7,14-16).

There remains controversy regarding whether malignant myoepitheliomas and spindle cell carcinomas share the same histogenesis, as both neoplasms exhibit a biphasic growth pattern. It is hypothesized that both elements (mesenchymal and epithelial) are derived from a common cell of origin or a pluripotent stem cell, although this hypothesis has not yet been confirmed $(3,6,7)$. Despite extensive sampling, concurrent presence of ordinary intraductal or invasive ductal carcinoma or foci of other types of metaplastic differentiation (e.g., squamoid, chondroid or osseous) were not observed. These are common characteristics of metaplastic spindle cell carcinomas. The diagnosis is further confirmed using antibodies against CKs of variable molecular weight. Both types of neoplasms express p63 and CK5, but the presence of smooth-muscle markers and immunostaining for GFAP favors myoepithelial differentiation. Metaplastic sarcomatoid carcinomas are usually reactive for CK7, CK5/6, CK903 and CKAE1-3.

Primary spindle-cell sarcomas, such as fibrosarcomas and dediferentiated liposarcomas, are generally not immunoreactive for CKs. Leiomyosarcomas and rhabdomyosarcomas stain diffusely and strongly positive with muscle markers, such as SMA, desmin and caldesmon, or myoglobin, myogenin and myo-D1, respectively.
Fibromatosis should also be excluded prior to confirming the diagnosis of myoepithelioma. Fibromatosis is a well-recognized proliferation of myofibroblasts and fibroblasts intermingled with abundant stromal collagen. Minimal pleomorphism and the absence or minimal number of mitoses are indicative of the benign nature of this lesion. Immunohistochemically, fibromatosis often expresses actin, occasionally desmin and S-100, while CD34 and CKs are not expressed $(6,7,14,15)$.

Other neoplasms that should be included in the differential diagnosis are myofribroblastoma (usually negative for $\mathrm{CKs}$ and S100 and positive for smooth muscle myosin-heavy chain and CD34), solitary fibrous tumor, sclerosing adenosis with prominent myoepithelial cells (usually in the elderly, associated with atrophy of the epithelial component), melanoma, malignant fibrous histiocytoma and malignant peripheral nerve sheath tumor $(7,14,15)$.

Due to the rarity of myoepithelial carcinoma and the limited data availability, very little is known on its biological behavior and systemic treatment.

By the time the patient was admitted to our medical center, she already had advanced-stage (IIIb) breast cancer with inflammation/ulceration of the nipple and skin, involvement of the chest wall and metastasis to axillary lymph nodes, but no distant metastatic disease. Neoadjuvant chemotherapy with docetaxel, doxorubicin and cyclophosphamide was administered for cytoreduction in order to render the tumor operable. Chemotherapy not only proved ineffective in that respect, but the CT scans also revealed locoregional progression of the disease and the development of an abscess in the tumor area. Therefore, a palliative simple mastectomy was performed and further chemotherapy was scheduled to treat the residual disease.

Taking into consideration the unresponsiveness of the tumor to neoadjuvant therapy, there was an alteration of regimens postoperatively. The patient was treated with paclitaxel, carboplatin and bevacizumab. Despite the administration of chemotherapy, a locoregional relapse occurred soon after surgery, which emphasizes the agressiveness of the tumor and its resistance to treatment.

Myoepithelial carcinoma has an aggressive course, with locally invasive and widespread metastatic potential to several organs, commonly the liver and lungs, and the tendency to recur, even with clear resection margins. The treatment of choice is surgical, namely wide tumor excision or mastectomy with regional lymph node excision $(5,10,17,18)$.

Adjuvant radiotherapy $(1,19-21)$ or chemoradiation $(22,23)$ are often administered in order to minimize local recurrence.

The effectiveness of hormonal therapy is unknown $(4,9)$. Since the tumor was triple-negative on IHC, which is characteristic of malignant myoepitheliomas, we considered that hormonal therapy would be of no benefit to the patient.

There are not sufficient data available to define the role and effectiveness of first-line chemotherapy, although it remains the only therapeutic choice in cases with distant metastasis or recurrence disease. However, none of the regimens used thus far have been proven effective (including carboplatin, paclitaxel, doxorubicin, cyclophosphamide, gemcitabine and oral capecitabine) (3). A multidisciplinary treatment approach is recommended, taking into account all the particularities of this rare type of cancer and its ambiguous biological behavior. 


\section{Acknowledgements}

The authors would like to thank Mr. Kostas P. Karamoustos for his valuable support in connection with the technical processing of all the photos which are included in this project.

\section{References}

1. Liao KC, Lee WY and Chen MJ: Myoepithelial carcinoma: A rare neoplasm of the breast. Breast Care (Basel) 5: 246-249, 2010.

2. Ahmed A: The myoepithelium in human breast carcinoma. J Pathol 113: 129-135, 1974.

3. Endo Y, Sugiura H, Yamashita H, Takahashi S, Yoshimoto N Iwasa M, Asano T and Toyama T: Myoepithelial carcinoma of the breast treated with surgery and chemotherapy. Case Rep Oncol Med 2013: 164761, 2013.

4. Lakhani SR, O'Hare MJ, Monaghan P, Winehouse J, Gazet JC and Sloane JP: Malignant myoepithelioma (myoepithelial carcinoma) of the breast: A detailed cytokeratin study. J Clin Pathol 48: 164-167, 1995.

5. Liang YF, Zeng JC, Ruan JB, Kang DP, Wang LM, Chen C, $\mathrm{Xu} \mathrm{JF}$ and Wu QL: Malignant myoepithelioma of the breast: A case report and review of literature. Int J Clin Exp Pathol 7: 2654-2657, 2014

6. Tavassoli FA: Pathology of the Breast. 2nd edition. Appleton and Lange, Stamford, CT, pp763-791, 1999.

7. Hoda SA, Brogi E, Koernen FC and Rosen PP (eds): Rosen's Breast Pathology. 4th edition. Lippincott Williams \& Wilkins, Philadelphia, PA, pp174-180, 2014.

8. Lakhani SR, Ellis IO, Schnitt SJ, Tan PH, van de Vijrer MJ (eds): WHO Classification of Tumours of the Breast.IARC Press, Lyon, France, pp120-123, 2012.

9. Suguna BV, Geethamani V, Niveditha SR and Monika LS: Malignant myoepithelioma of the breast: Case report with review of literature. Malaysian J Pathol 33: 125-128, 2011.

10. Poret H, Cormier B, Marret H, Body G and Ouldamer L: Malignant myoepithelial breast carcinoma: Diagnosis and therapeutic difficulties. Gynecol Obstet Fertil 41: 334-337, 2013.

11. Fayaz S, Samir S, Amanguno H, El-Sherify M, Adesina A, Abozlouf S, George T, Attia G, Eissa H and Bedair A: Myoepithelioma (myoepithelial carcinoma) of the breast: Case reports. Austral-Asian Journal of Cancer 12: 7-12, 2013.
12. Enghardt MH and Hale JH: An epithelial and spindle cell breast tumour of myoepithelial origin. An immunohistochemical and ultrastructural study. Virchows Arch A Pathol Anat Histopathol 416: 177-184, 1989.

13. Lingamfelter D, Chen Y, Kure $\mathrm{K}$ and Lankachandra K: Infiltrating myoepithelial carcinoma of the breast, a case report and cytologic-histologic correlation. Diagnostic Pathology 3: 7, 2008.

14. Dabbs DJ: Breast Pathology. 1st edition. Elsevier Saunders, Philadelphia, PA, pp307-318; 479-490, 2012.

15. Dabbs DJ: Diagnostic Immunohistochemistry: Theranostic and Genomic Application. 3rd edition, Saunders Elsevier, Philadelphia, PA, pp102-109; 783-785, 2010.

16. Gattuso P, Reddy VB and D Odile: Differential Diagnosis in Surgical Pathology: Expert Consult-Online and print, 2e. 2nd edition. Saunders, 2009.

17. Khattech R, Ben Othman M, Ben Romdhane K, Gamoudi A and Ammar A: Breast myoepithelioma. Report of a case. Ann Pathol 15: 138-141, 1995 (In French).

18. Brasseur P, Hustin J and Collard M: Breast tumor with myoepithelial cells. Apropos of a case report. J Belge Radiol 73: 197-200, 1990 (In French).

19. Scarpellini F, Usellini L and Foschini MP: Malignant myoepithelioma associated with in situ and invasive ductal carcinoma. Description of a case and review of the literature. Pathologica 89: 420-424, 1997 (In Italian).

20. Loose JH, Patchefsky AS, Hollander IJ, Lavin LS, Cooper HS and Katz SM: Adenomyoepithelioma of the breast. A spectrum of biologic behavior. Am J Surg Pathol 16: 168-876, 1992.

21. Fang ZM, Tse RV, Marjoniemi VM, Kozlov S, Lavin MF, Chen H, Kearsley JH, Graham PH and Clarke RA: Radioresistant malignant myoepithelioma of the breast with high level of ataxia telangiectasia mutated protein. J Med Imaging Radiat Oncol 53: 234-239, 2009.

22. Terada T: Malignant myoepithelioma of the breast. Pathol Int 61: 99-103, 2011.

23. Trepp R, Padberg BC, Varga Z, Cathomas R, Inauen R and Reinhart WH: Extensive extranodal metastases of basal-like breast cancer with predominant myoepithelial spindle cell differentiation. Pathol Res Pract 206: 334-337, 2010. 